\title{
Influence d'une présure d'agneau sur la qualité du fromage Kefalotyri
}

\author{
par \\ le Dr Emmanuel ANIFANTAKIS \\ Maître-Assistant à la chaire de Technologie Laitière \\ de l'Ecole Supérieure d'Agriculture d'Athènes (Grèce)
}

\section{I. - INTRODUCTION}

En Grèce, dans un certain nombre de fromageries permanentes ou rudimentaires, on utilise pour la coagulation du lait, une présure faite d'une manière empirique, à partir de caillettes d'agneau. On a remarqué que cette présure, souvent mélangée avec un certain pourcentage de présure en poudre, donne une saveur piquante aux fromages, qualité pour laquelle les consommateurs grecs montrent une certaine préférence.

Malgré que dans le commerce on ne trouve pas à acheter cette présure, connue comme "présure locale », une certaine quantité, préparée d'une manière artisanale, est utilisée pour la préparation des fromages faits, par principe, avec du lait de brebis. Cette présure contient soit la totalité des caillettes d'agneaux ou de chevreaux, soit le lait coagulé qui se trouve dans les caillettes et, plus rarement, seulement leurs tissus.

Pour éviter les contaminations, que parfois on rencontre dans les présures artisanales et qui posent des problèmes à la fabrication, nous avons préparé cette présure sous forme de poudre que nous avons utilisée pour fabriquer le fromage Kefalotyri et étudier l'influence sur ses qualités physico-chimiques et organoleptiques et, par conséquent, convenable pour l'industrie.

Il faut aussi considérer un autre aspect du problème : selon les données du Service Statistique de Grèce (1974), nous avons une consommation de viande de 5,4 millions d'agneaux et 3,1 millions de chevreaux par an. On utilise alors une quantité de leurs caillettes pour la préparation des présures artisanales, dont on se sert pour la fabrication traditionnelle de fromages Feta et Kefálotyri, comme remède à la pénurie mondiale de caillettes de veau. 
On peut se demander alors si l'étude de la préparation et les qualités de cette présure n'est pas nécessaire pour éviter le gaspillage d'une matière première si précieuse puisque la présure devient de plus en plus rare.

Dans une de nos études (Anifantakis, 1975), nous avons étudié largement la préparation de cette présure ainsi que ses qualités physico-chimiques, et les résultats obtenus ont été très favorables.

Tenant compte de l'importance du sujet, la présente recherche est la première d'une série pour préciser l'importance de la présure, faite à partir de caillettes d'agneau, sur les qualités physicochimiques et organoleptiques des fromages grecs traditionnels. Nos résultats permettront de savoir si ce nouveau produit est utile pour la fabrication des fromages grecs et, par conséquent, s'il faut construire une usine pour la préparation de cette présure.

\section{II. - PARTIE EXPERIMENTALE}

\section{1) Matériel et méthodes}

Malgré qu'en Grèce le fromage Kefalotyri soit préparé par principe avec du lait de brebis, nous avons dans nos essais utilisé le lait de vache, étant donné que dernièrement nous avons eu une pénurie de lait de brebis.

Le lait de vache que nous avons utilisé était d'une bonne qualité bactériologique, exempt d'antibiotiques, d'une acidité de $15-16^{\circ} \mathrm{D}$ et titrant 3,2 p. 100 de matière grasse. Comme présure témoin nous avons utilisé la présure liquide de Hansen.

Nous avons préparé notre présure en partant de caillettes d'agneau découpées en lanières, séchées, et traitées selon un mode de préparation que nous avons déjà étudié (Anifantakis, 1975). On a obtenu le produit final en poudre. A cause des résidus de caillettes dans la poudre, sa dilution dans l'eau n'était pas limpide mais se dispersait de façon égale dans le lait.

\section{2) Technologie}

La technologie utilisée pour la préparation de fromage Kefalotyri, comme pour la plupart des fromages grecs, est le résultat d'un empirisme transmis de génération en génération et de région en région, et ce qu'il faut constater est qu'elle n'est pas standardisée et que, par conséquent, les produits ne sont pas d'une composition définie. Ainsi, dans le commerce on trouve le fromage Kefalotyri sous différents noms locaux, Crête, Thessalie, etc., et pour cela la composition et les caractères organoleptiques sont différents (Zigouris, 
1952). Dans nos essais, nous avons utilisé la technologie recommandée par le laboratoire de Technologie laitière de l'Ecole supérieure d'agriculture d'Athènes.

La pasteurisation était faite dans une cuve à double paroi à $68^{\circ} \mathrm{C}$ pendant $10 \mathrm{mn}$, et ensuite le lait était refroidi à $34^{\circ} \mathrm{C}$.

Comme ferments lactiques nous avons ajouté Streptococcus lactis et cremoris dans un pourcentage de 1 p. 100, puis Lact. bulgaricus et Str. thermophilus à $1 \mathrm{p}$. 1000. La quantité de présure ajoutée était suffisante pour avoir un temps de prise de $12-14 \mathrm{mn}(10,8 \mathrm{ml}$ de présure liquide de Hansen et $15 \mathrm{~g}$ de poudre de présure d'agneau pour 1001 de lait).

La technologie que nous avons suivie pour la préparation du fromage Kefalotyri peut être résumée comme suit :

Premier jour :

8.00 a.m. - Pasteurisation du lait et refroidissement à $34^{\circ} \mathrm{C}$, addition des ferments.

8.17 - Addition de la présure liquide Hansen, 10,8 ml, présure d'agneau, $15 \mathrm{~g}$.

$8.30-$ Temps de prise.

8.55 - Découpage immédiatement après la coagulation, en grains d'une grandeur du grain de blé.

$9.00-$ Fin du découpage.

9.15 - Brassage.

$9.30-$ Réchauffement du caillé jusqu'à $46^{\circ} \mathrm{C}-47^{\circ} \mathrm{C}$.

9.45 - Brassage.

9.50 - Repos.

9.55 - Pressage des grains à la main sur toute la surface de la cuve. Dès que les grains étaient collés, le fromager faisait un rouleau avec le caillé dans le lactosérum pour obtenir finalement un cylindre. Il coupait ensuite ce cylindre en morceaux, qu'il transportait dans les moules. Après malaxage à la main les fromages étaient pressés pendant $5 \mathrm{~h}$.

15.00 - Les fromages, dans les moules, étaient transportés dans une cave à $12^{\circ} \mathrm{C}-14^{\circ} \mathrm{C}$.

\section{Troisième jour :}

De la cave à $12^{\circ} \mathrm{C}-14^{\circ} \mathrm{C}$, les fromages ont été placés en saumure de $18-20^{\circ}$ Bé, à une température de $12^{\circ} \mathrm{C}-14^{\circ} \mathrm{C}$ pendant $24 \mathrm{~h}$. Le salage continuait avec du gros sel sur tous les côtés, chaque $2 \mathrm{j}$, et pendant les 40 premiers jours. Temps d'affinage 3 mois. 


\section{3) Analyses}

Pour arriver à la même fermeté du caillé dans tous les cas, nous avons utilisé deux torsiomètres de Scott-Blair (1963), d'une échelle de $0-420^{\circ}$.

Les fromages affinés, après 3 mois ont été examinés en ce qui concerne l'azote soluble et non soluble, selon la méthode Kjeldahl (A.O.A.C. 1960), tandis que les acides gras libres étaient estimés selon la méthode Harper (1953).

Une dégustation des fromages affinés a eu lieu par un groupe du personnel du laboratoire, pour apprécier leur qualité organoleptique et remarquer s'il existait une différence qualitative, surtout en ce qui concerne la saveur piquante.

\section{III. - RESULTATS}

On remarque que le but de la présente recherche n'était pas l'étude de la technologie du fromage Kefalotyri, mais la recherche de la possibilité de l'utilisation de la présure d'agneau pour constater éventuellement certains de ses inconvénients ou avantages. La technologie, quoique empirique, était utilisée pour la préparation des

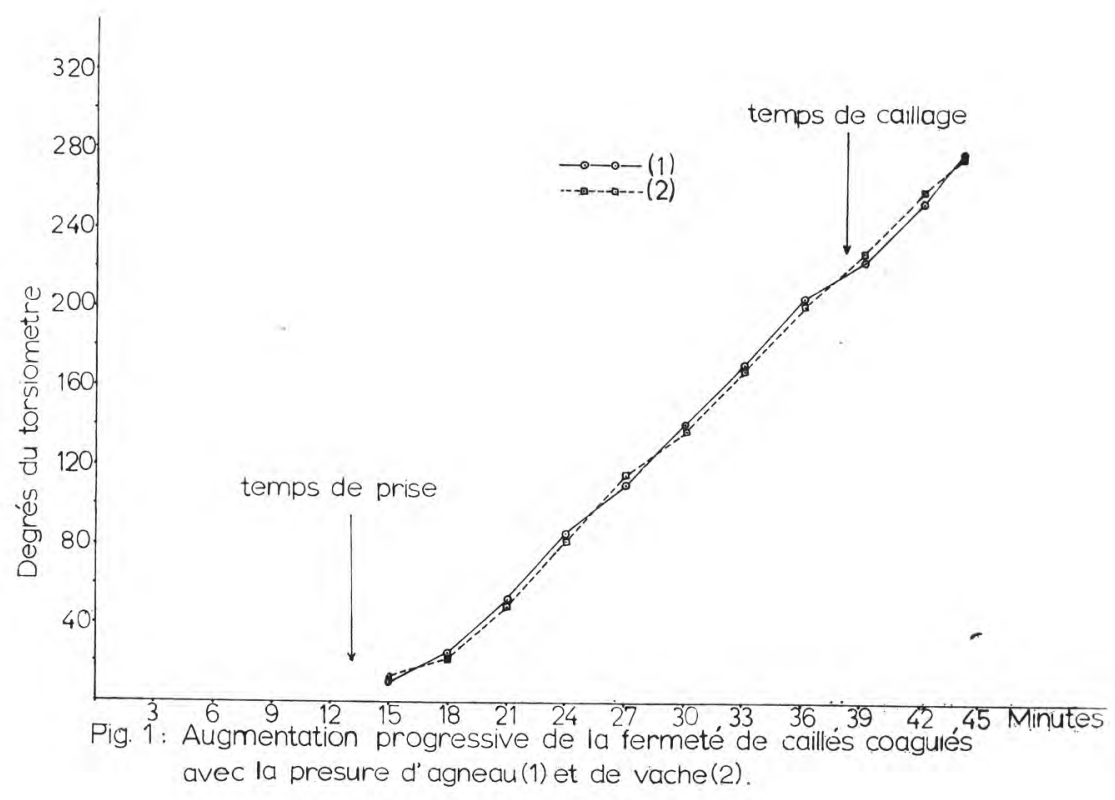

fig. 1 
fromages afin de montrer s'il existait une différence de comportement des deux présures.

D'abord, on a étudié si la vitesse de l'augmentation de la fermeté était différente avec l'utilisation des deux différentes présures. Il faut noter que pareille différence a été trouvée entre la présure Hansen et celle de Pfizer (Kalatzopoulos, 1970).

Dans une série d'essais, en même temps que la fabrication du fromage, on a étudié les qualités rhéologiques du caillé à l'aide de deux torsiomètres Scott-Blair (1963). La figure 1 montre l'augmentation progressive de la fermeté des caillés coagulés avec la présure d'agneau (1) et celle de vache (2), en quantités équivalentes (temps de prise $12-14 \mathrm{mn}$ ). Le découpage du caillé a été fait empiriquement à un degré de fermeté compris entre 200 et $220^{\circ}$ du torsiomètre.

Le degré de maturation des fromages préparés, après 3 mois, a été calculé par le coefficient de maturation. Le coefficient de maturation est la quantité d'azote soluble dans le fromage, exprimée en pourcentage de l'azote total (tab. 1). Des données du tableau 1 nous pouvons constater qu'il existe des différences dans les valeurs du coefficient de maturation des deux sortes de présure, mais qui ne sont pas statistiquement valables pour une probabilité de 5 p. 100 . Etant donné que les essais ont eu lieu sous les mêmes conditions, nous pouvons conclure que nous avons le même degré de protéolyse en fin de maturation des fromages.

Les résultats obtenus ont une valeur indicative, car le degré de protéolyse n'est pas le fait seul des présures utilisées, mais aussi des micro-organismes. Le calcul de la contribution de chacun d'eux est trop pénible et coûteux, étant donné que les essais doivent avoir lieu sous conditions d'asepsie.

Il est bien connu qu'à la formation du goût caractéristique des fromages, prennent part aussi les acides gras libres. La majorité d'entre eux provient de l'hydrolyse de la matière grasse du lait, tandis qu'une partie moins importante est due à la fermentation du lactose et à la transformation des acides aminés.

Le degré d'action des enzymes lipolytiques au cours de la maturation a une influence sur les produits d'hydrolyse de la matière grasse. Il faut remarquer qu'une pareille action n'est pas désirable et selon la F.I.L. (1973), des produits de remplacement de la présure de veau ayant une action lipolytique notable, ne sont pas utilisables.

La présure d'agneau, comme nous l'avons déjà constaté (Anifantakis, 1975), a une action faible sur la tributyrine, mais pas sur la matière grasse du lait. Dans ce dernier cas nous ne pouvons pas distinguer si la présure d'agneau a une action lipolytique faible, en raison des conditions de notre travail, mais elle sera peut-être notable après maturation du fromage, avec apparition d'un goût piquant. 
TABLEAU 1. - Degré de maturation des fromages après 3 mois

\begin{tabular}{|c|c|c|c|c|c|c|c|}
\hline \multirow{2}{*}{ Analyses effectuées } & \multicolumn{3}{|c|}{ Présure Hansen } & \multicolumn{3}{|c|}{ Présure d'agneau } & \multirow{2}{*}{$\begin{array}{c}\mathrm{T} \\
\text { probab. } \\
5 \text { p. } 100\end{array}$} \\
\hline & moyen & $\begin{array}{l}\text { intervalle } \\
\text { de prix }\end{array}$ & $\begin{array}{l}\text { écart } \\
\text { type }\end{array}$ & moyen & $\begin{array}{l}\text { intervalle } \\
\text { de prix }\end{array}$ & $\begin{array}{l}\text { écart } \\
\text { type }\end{array}$ & \\
\hline $\mathrm{N}$ total p. 100 & $3, \dot{84}$ & $3,71-3,98$ & 0,09 & 3,87 & $3,74-4,02$ & 0,1 & 1,19 \\
\hline $\mathrm{N}$ soluble p. 100 & 0,83 & $0,69-0,92$ & 0,08 & 0,84 & $0,74-0,94$ & 0,09 & 0,05 \\
\hline Coefficient de maturation & 21,40 & $18,4-24$ & 2,25 & 22,00 & $19,3-24,6$ & 2,29 & 0,25 \\
\hline
\end{tabular}

TABLEAU 2

Acides gras libres du fromage Kefalotyri après 3 mois ( $\mu$ moles $/ 5 \mathrm{~g}$ de fromage)

\begin{tabular}{|c|c|c|c|c|c|c|c|}
\hline \multirow{2}{*}{ Acides gras } & \multicolumn{3}{|c|}{ Présure Hansen } & \multicolumn{3}{|c|}{ Présure d'agneau } & \multirow{2}{*}{$\begin{array}{c}\mathrm{T} \\
\text { probab } \\
5 \mathrm{p} .100\end{array}$} \\
\hline & moyen & $\begin{array}{l}\text { intervalle } \\
\text { de prix }\end{array}$ & $\begin{array}{l}\text { écart } \\
\text { type }\end{array}$ & moyen & $\begin{array}{l}\text { intervalle } \\
\text { de prix }\end{array}$ & $\begin{array}{l}\text { écart } \\
\text { type }\end{array}$ & \\
\hline $\mathrm{C}_{2}$ & 6,75 & $5,4-7,8$ & 0,98 & 8,45 & $7,3-10,7$ & 1,46 & $4,304^{*}$ \\
\hline $\mathrm{C}_{3}$ & 1,96 & $1,0-3,0$ & 0,65 & 3,30 & $2,8-4,8$ & 0,83 & $4,278^{*}$ \\
\hline $\mathrm{C}_{4}$ & 17,55 & $13,6-21,6$ & 3,13 & 20,21 & $16,4-25,1$ & 3,31 & $14,651^{*}$ \\
\hline $\mathrm{C}_{4}$ & 59,20 & $53,8-63,7$ & 3,40 & 63,66 & $57,6-69,3$ & 3,77 & $8,316^{*}$ \\
\hline
\end{tabular}


Pour vérifier une pareille hypothèse nous avons déterminé, par chromatographie sur colonne, selon la méthode de Harper (1953), les acides gras libres dans les fromages, après 3 mois, et les résultats sont présentés dans le tableau 2. De ce dernier tableau nous pouvons constater que la quantité d'acides gras libres dans le cas des fromages préparés en utilisant de la présure d'agneau est plus importante, sans savoir s'ils sont responsables des qualités organoleptiques des fromages (saveur piquante).

Pour vérifier si les acides différents avaient une influence sur le goût on a utilisé un groupe de dix-neuf personnes du laboratoire comme dégustateurs. L'échelle proposée était :
qualité très bonne 5-6
qualité bonne $\quad 3-5$
qualité médiocre 3-1
qualité mauvaise 0

Les résultats des examens organoleptiques ont montré que tous les fromages étaient d'une bonne qualité : 3,8 pour ceux qui étaient coagulés avec la présure d'agneau et 4,1 pour ceux faits avec la présure de veau.

Les dégustateurs ont remarqué le goût piquant dans le cas des fromages préparés avec la présure d'agneau, qualité pour laquelle les consommateurs grecs montrent une certaine préférence. Comme nous l'avons déjà signalé, ce goût provient des enzymes lipolytiques que contient la présure d'agneau, donc l'action sur la tributyrine a été démontrée.

Bien que nous n'ayons pas examiné les fromages après 3 mois, nous pouvons dire que pour un temps de maturation plus long le goût piquant sera plus fort. Pour le consommateur grec cette " qualité " est un avantage et comme tous les fromages étaient estimés négociables, la présure d'agneau se révèle être un produit intéressant pour notre Pays.

\section{CONCLUSION}

Une présure préparée à partir de caillettes d'agneau a été utilisée avec succès pour la préparation du fromage Kefalotyri. On a montré que son utilisation ne pose pas de problèmes ni à la technologie, ni à la maturation, nous avons trouvé le même coefficient de maturation contre les témoins, fromages préparés avec la présure Hansen.

Le produit final a les caractères organoleptiques du fromage préparé avec la présure traditionnelle. De plus, les fromages faits avec la présure d'agneau ont une saveur piquante que les consommateurs grecs préfèrent.

Cette dernière qualité provient des enzymes lipolytiques que la présure d'agneau contient et grâce auxquelles une quantité importante des acides gras est libérée. 


\section{S u m m a r y}

After a short litterature review, the methods and materials used are described for the study of the effect that sheep's rennet has on the quality of the Kefalotyri cheese. The obtained results are discussed and conclusions are drawn, which in short are the following :

For the production of Kefalotyri cheese, sheep's rennet could be used successfully as a substitute for the traditional one. It was observed that no significant change is taking place in the practical technology of the said cheese. With the coefficient of maturity as criterion, the rate of maturity is almost similar of the traditional cheese. The mature cheese is marketable and has similar organoleptic characteristics with the cheese made by the traditional rennet, but the first has peppery taste.

A statistically significant difference exists between the two cheese in the free fatty acids concentration, with the cheese made by sheep's rennet having always the higher.

\section{Remerciements}

Nous remercions $M$. G. Kalatzopoulos pour ses suggestions et ses critiques au cours de la rédaction du manuscrit.

\section{Références bibliographiques}

1. Anifantakis (Emm.) (1975). - Contribution à l'étude d'une présure faite à partir des caillettes d'agneau et chevreau. Thèse d'agrégation.

2. BerRidge (N. J.) (1943). - Food Manufacture, 13, 305-308.

3. Bulletin du Service Statistique de Grèce (1974).

4. Fédération Internationale de Laiterie (1973). - Bulletin annuel, $\mathrm{n}^{\circ} 74$.

5. Foltmann (B.) (1966). - Compt. rend. des trav. Lab. Carlbsberg.

6. HARPER (M. J.) (1953). - J.D.SC., 7, 808.

7. Kalatzopoulos (G.) (1970). - Thèse.

8. Official Method of Analyse A.O.A.C. (1960).

9. Scott-Blair (G.) (1963). - Dairy Ind., p. 220. 